\title{
L'initiation à la recherche par la thématique des ambiances
}

An Introduction to Research Through the Theme of Ambiances

Pascal Joanne, Thomas Ouard et Nathalie Simonnot

\section{(2) OpenEdition}

Édition électronique

URL : http://journals.openedition.org/crau/529

DOI : $10.4000 /$ crau.529

ISSN : 2547-5746

Éditeur

Éditions du patrimoine

Édition imprimée

Date de publication : 1 novembre 2012

Pagination : 24-30

ISBN : 978-2-7577-0108-9

ISSN : 1296-4077

Référence électronique

Pascal Joanne, Thomas Ouard et Nathalie Simonnot, « L'initiation à la recherche par la thématique des ambiances », Les Cahiers de la recherche architecturale et urbaine [En ligne], 26/27 | 2012, mis en ligne le 01 novembre 2017, consulté le 10 décembre 2020. URL : http://journals.openedition.org/crau/529 ; DOI : https://doi.org/10.4000/crau.529 
L'objectif du mémoire $d$ 'initiation à la recherche durant la première année du cycle master, réside dans l'apprentissage et la mise en œuvre de méthodes appropriées, et dans la compréhension qu'un sujet reste inerte s'il n'est pas interrogé de manière dynamique. La thématique des ambiances, avec la grande hétérogénéité de ses champs disciplinaires, peut être le vecteur de ces questionnements. Son périmètre mouvant, sans cesse redéfini et réinterrogé, constitue un puissant stimulant à l'exploration de mises en perspective originales.

\section{L'initiation à la recherche par la thématique des ambiances}

PASCAL JOANNE THOMAS OUARD NATHALIE SIMONNOT

Au cours du cycle master conduisant au diplôme d'État d'architecte, tout étudiant inscrit dans une école nationale supérieure d'architecture doit réaliser un mémoire censé attester de sa capacité à produire un travail d'investigation intellectuel sur un sujet donné. Selon les textes réglementaires ${ }^{1}$, « le mémoire est un travail personnel d'études et (ou) de recherche qui permet à l'étudiant de traiter une problématique propre à un séminaire ou à un travail en lien avec le projet ». Dans le cadre de l'un des quatre domaines d'étude proposés durant le cycle master à l'école nationale supérieure d'architecture de Nantes et intitulé "Cultures constructives et stratégies de projet», le séminaire "Thématique des ambiances ${ }^{2}$ ", propose aux étudiants de travailler autour de «l'expérience sensible d'un sujet dans l'espacetemps, d'une pratique habitante ou citadine ${ }^{3} »$. Réalisé pendant les deux premiers semestres du master, le travail est validé en deux temps : d'abord par l'évaluation d'une note méthodologique destinée à rendre compte de la formulation d'une problématique, ensuite par la rédaction du mémoire proprement dit et de la mise

1. Arrêté du 20 juillet 2005 relatif aux cycles de formation des études d'architecture conduisant au diplôme d'études en architecture conférant le grade de licence et au diplôme d'État d'architecte conférant le grade master, (NOR: MCCLO500496A), articles 17 et 18. Journal officiel n 199 du 27 août 2005, page 13944 , texte $n^{\circ} 59$
2. Cette notion d'ambiance est explorée depuis maintenant plusieurs années par des équipes de recherche comme le CRESSON de l'école nationale supérieure d'architecture de Grenoble et le CERMA de l'école nationale supérieure d'architecture de Nantes. Le CERMa, Centre de recherche méthodologique d'architecture, constitue avec le laboratoire CRESSON, Centre de recherche sur l'espace sonore et l'environnement urbain, 
en œuvre de la méthodologie choisie pour répondre à la problématique.

Le mémoire d'initiation à la recherche n'a pas pour but de former des chercheurs, bien qu'il puisse aussi révéler les appétences et les capacités de quelques étudiants à la recherche. Ces derniers poursuivront éventuellement leur parcours avec une mention recherche, voire pour certains vers une inscription en doctorat en constituant les forces vives de la recherche en architecture dans les laboratoires des écoles. Cet exercice doit être considéré comme une activité intellectuelle à part entière nécessaire à tout architecte diplômé quelle que soit la profession qu'il exercera, d'où son caractère obligatoire dans la formation.

La construction du mémoire relève, à notre sens, de la compréhension de trois entités distinctes : la définition d'un sujet, la formulation d'une problématique et la mise au point d'une méthode appropriée. En cela, cette manière de procéder est identique à de nombreux mémoires de recherche, quelle qu'en soit la thématique. La « jeune recherche en architecture » se forme ainsi au contact de cet apprentissage, volontairement exigeant dans la distinction de ces trois entités. L'objectif même du mémoire est de savoir se détacher d'un sujet en le mettant à distance grâce à une approche critique et de tendre vers une certaine objectivité. Ce détachement est d'autant plus nécessaire que l'étudiant est " attaché » à son sujet. Le mémoire naît donc de la rencontre entre cette intrigue de départ et le souhait d'en maîtriser la complexité par un questionnement approprié. L'étudiant n'est pas seulement face à un problème ; il le crée et il cherche à le résoudre à travers la formulation d'une problématique de recherche.

\section{Définir un sujet : la remise à plat des certitudes}

Souvent considéré par les étudiants comme une épreuve difficile à laquelle ils sont confrontés pour la première fois, cet exercice trouve des résonances évidentes avec la pratique du projet d'architecture en raison de l'appareil intellectuel qu'il mobilise et de la démarche conceptuelle qui lui est associée. Selon Thierry Verdier, "la recherche [...] exprime la transcendance de la pensée à l'œuvre. Elle est " projet « au sens que l'on donne à ce mot en école d'architecture » et "le mémoire témoigne de la façon avec laquelle un étudiant parvient à fonder un jugement, ce qui, d'une certaine manière, est très proche de processus de création en architecture ${ }^{4}$ ". La parenté entre ces deux manières de concevoir un projet (au sens où le mémoire de recherche est projet) conditionne bien souvent le choix d'un sujet de mémoire. Intrigués par la complexité de l'ambiance d'un lieu ou par la perception d'un phénomène sensible dans un cadre architectural ou urbain, l'étudiant choisit de s'investir dans une réflexion de fond lui permettant de décomposer et d'analyser avec une méthode appropriée les tenants et les aboutissants de cette interrogation. Aussi, beaucoup de sujets proposés (par les étudiants, le plus souvent) sont en relation avec des questionnements actuels et/ou avec la pratique du projet. Cette démarche est rendue d'autant plus difficile que cette interrogation est en prise immédiate avec le monde contemporain dans lequel la distance critique nécessaire à toute analyse est difficile à mettre en œuvre.
I'unité mixte de recherche CNRS 1563

Ambiances architecturales et urbaines.

3. Voir le «Livret de l'étudiant 2009-2010", Énsa Nantes, p. 130.
4. Thierry Verdier, «Guide pour la rédaction d'un mémoire en architecture ", Carnets de la recherche, hors série, Montpellier, Éditions de l'Espérou, juin 2009, p. 8. 
L'une des spécificités du mémoire d'initiation à la recherche dans le champ des ambiances est d'ouvrir à des questionnements originaux construits, soit autour de la fabrication de l'ambiance, soit autour de sa perception. L'étudiant n'a pas conscience de la catégorisation de cette approche lorsqu'il définit un sujet. L'intérêt de départ qui est manifesté au moment où l'étudiant prend contact avec l'équipe pédagogique pour travailler sur les ambiances, est concentré autour de sujets aux contours très larges: le développement durable, la lumière, les éco-quartiers, etc. L'intérêt pour ces domaines de recherche s'explique par les problématiques développées dans l'exercice du projet et son lien avec la demande sociale et économique. Parmi les sujets proposés, une étude statistique basée sur les mémoires produits entre 2007 et $2010^{5}$ dans le séminaire sur les ambiances (une quarantaine de travaux), montre que plus de la moitié sont consacrés à la conception et aux usages du projet architectural, à l'actualité du développement durable et aux ambiances liées aux éléments naturels (eau, végétal). Le reste des mémoires porte sur des manières de percevoir (les sens sollicités), des types et des formes d'espaces, des modes de représentation, dans un lien plus distant avec le projet architectural. L'habitat (individuel, collectif) constitue souvent la porte d'entrée de ces recherches et montre que la plupart des mémoires sont centrés sur l'échelle architecturale. On observe, néanmoins, que le sujet des éco-quartiers et de leurs aménagements apparaît de manière récurrente. La dimension urbaine est souvent présente dans les propositions de départ car elle donne l'illusion d'être une source de question- nements plus larges que la seule échelle architecturale. L'expérience montre, toutefois, que la précision gagnée au fur et à mesure de l'avancée du mémoire dans la définition de la problématique, recentre en général le propos à une échelle plus réduite où les questionnements sont moins nombreux mais gagnent en pertinence et en subtilité.

La construction de l'intérêt passe donc par une phase d'appropriation du sujet et par un élagage nécessaire permettant de préciser le contenu. Beaucoup de sujets mal définis ou trop ancrés dans la fabrication du projet architectural, peuvent en quelques semaines être croisés avec un questionnement d'ambiance. Des sujets centrés autour de monographies de bâtiments gagnent à être analysés sous l'angle d'un facteur d'ambiance, d'un mode de représentation ou d'une forme de perception. Pendant cette phase de définition du sujet de mémoire, l'étudiant est souvent amené à réinterrogerles mots et leur signification. C'est la première étape vers la déconstruction des certitudes, un moment de remise à plat des termes et de leur relation. Un mémoire portant sur les ambiances dans les éco-quartiers ${ }^{6}$ lors de la phase d'aménagement et de concertation entre les partenaires du projet, a nécessité d'interroger la signification même du mot " éco-quartier » en faisant apparaître une diversité d'acceptions, alors que le terme semblait aller de soi pour l'étudiant qui entendait entrer immédiatement dans l'analyse des ambiances. Le sujet se construit ainsi dans la définition d'un périmètre de recherche qui, seul, pourra permettre de formuler dans un second temps une problématique claire. Les mémoires dont la problématique peine à émerger ou
5. Séminaire dirigé par Pascal Joanne, Thomas Ouard et Nathalie Simonnot durant cette période.
6. Éric Ridou, La prise en compte des ambiances dans les éco-quartiers, Énsa Nantes, 2009. 
dans lesquels elle est absente sont souvent le résultat d'une difficulté à avoir su préciser, au départ, l'exactitude d'un champ d'investigation.

\section{Construire une problématique : une question de prise de conscience}

Le sujet une fois défini n'est pas une fin en soi, même s'il constitue une étape incontournable. C'est la construction d'une démarche qui est évaluée et la capacité de l'étudiant à interroger, puis à articuler des modes de représentation, des références, des simulations ou des enquêtes par une méthode adéquate. En cela, le mémoire sur les ambiances constitue bien un objet de recherche à part entière, doté de ses thématiques et de ses méthodes propres. L'analyse d'un contexte, sa compréhension et la mise en évidence de questionnements ne diffèrent guère de la manière d'analyser un site dans le projet architectural ou urbain. La difficulté du mémoire de recherche est de n'être ni une synthèse (compilation de données), ni une étude (analyse de données). Tout au contraire, la synthèse et l'étude sont les outils de production de la recherche. Seule la formulation d'une problématique - qu'est-ce qui intrigue et comment peut-on y répondre ? - permet de comprendre l'intérêt et les objectifs de la recherche.

La compréhension des objectifs du mémoire de recherche relève d'une prise de conscience. La formulation d'une problématique montre la capacité à réunir un matériel intellectuel et à mettre en relation des éléments disparates. Quoi de plus insaisissable, subtil et complexe que de travailler sur l'ambiance? Comme " l'ambiance est probablement la chose au monde la plus facile à ressentir et la plus difficile à expliquer ${ }^{7}$ ", l'étudiant est amené à prendre conscience de l'intrication des phénomènes physiques (données objectives) avec leur perception sensible (données subjectives). La réflexion menée sur "les dimensions non visibles de l'espace construit ${ }^{8}$ " permet de développer des problématiques très riches à la confluence entre espaces conçus et espaces vécus. En travaillant sur la conception de la maison à basse consommation', un étudiant a mis en évidence l'importance de l'usage dans l'efficacité énergétique du projet. La maturité gagnée à l'énoncé de la problématique a permis de dépasser le sujet de départ défini autour de la seule phase amont du projet. L'étudiant pose ainsi I'hypothèse que la maison à énergie positive n'existe que si l'usage qui en est fait est contraint par un mode de fonctionnement particulier. L'efficience énergétique n'est donc pas seulement une affaire de conception du projet architectural (sujet de mémoire) mais de prise en compte d'une manière d'habiter (la problématique) mise en évidence grâce à une série d'entretiens avec les habitants (méthode). Un autre exemple montre comment le mémoire évolue favorablement de l'énoncé du sujet à la formulation de la problématique: intrigué par l'ambiance du village perçu comme une petite communauté rassurante, une étudiante a cherché à saisir les constituants de cette ambiance ${ }^{10}$. S'intéressant d'abord à l'aménagement de la place principale du village comme lieu d'identification de la communauté à un espace public de convergence (sujet), l'étudiante s'est intéressée à " l'esprit village » en montrant en quoi la notion d'identité communale est relative à la constitution d'un tissu social et associatif, bien avant d'être une simple affaire d'urbanisme (problé-
7. Jean-François Augoyard, «A comme Ambiance(s) », Les Cahiers de la recherche architecturale et urbaine $n^{\circ}$ 20/21, 2007, p. 33.

8. Ibid. p. 35.
9. Pierre-Edern Brulé, Développement durable et éco-construction : influence des modes de conception et d'habiter, Énsa Nantes, 2009, 146 p.

10. Charlotte Hubert, Esprit village, où es-tu? Énsa Nantes, 2009, 66 p. 
matique). Les échanges fructueux avec les habitants d'un village pris comme étude de cas (méthode) ont permis de vérifier quelques hypothèses.

Des sujets s'intéressent aux ambiances véhiculées par des représentations particulières de l'architecture par exemple l'usage de la photographie de bâtiments "à la tombée de la nuit"1", ou bien aux ambiances attachées à l'évolution des pratiques de l'espace public ${ }^{12}$. D'autres travaux plus théoriques interrogent la dimension référentielle $d u$ projet $d^{\prime}$ architecture ${ }^{13}$ ou encore, développent des questionnements autour de traités fondateurs et de leur réinterprétation dans des réalisations contemporaines. Prenons ce dernier exemple pour illustrer la démarche de recherche selon le déroulement temporel de la réflexion ${ }^{14}$ :

- le contexte et le sujet : I'étudiant s'intéresse à l'identité visuelle des bâtiments la nuit. La relation avec la thématique des ambiances est justifiée par la question de la perception nocturne. Dans un second temps, le sujet est défini et affiné en prenant appui sur la typologie du gratte-ciel (échelle architecturale) et sur les façades média lumineuses (un type d'existence nocturne) ;

- la problématique : partant d'un phénomène très contemporain et tout à fait spécifique, la problématique prend appui sur un questionnement théorique plus général, la thèse de la forme et de l'enseigne, développée par Robert Venturi dans Learning from Las Vegas paru en 1972 ;

- la méthode : la problématique, dès lors posée, nécessite le recours à une méthode de travail et la recherche de résultats pouvant corroborer ou infirmer le questionnement initial.
La question de la référence est commune à l'ensemble des mémoires, de manière plus ou moins évidente. Elle rend nécessaire la prise en compte d'un environnement culturel à partir duquel chacun se situe et, surtout, elle permet d'interroger de manière critique des sources de références (théorique, iconographique, langagière, symbolique...). L'incontournable relation avec la référence, consciente ou inconsciente, immédiate ou différée, évidente ou subtile, relève d'une prise de conscience d'un matériau existant à partir duquel chaque projet architectural ou urbain se situe. Lorsque le positionnement de cette référence est mis en évidence, une grande partie de la problématique a trouvé son sens.

\section{Obtenir des résultats : une question de méthode}

La mise au point du protocole permettant d'obtenir des résultats se fait dans le même temps que la construction de la problématique. L'un ne peut être pensé sans I'autre. Les méthodes mises au point sont de diverses natures et peuvent être divisées en deux groupes : celles qui produisent et analysent des données et celles qui analysent des données déjà existantes.

Dans le premier cas, les étudiants élaborent des entretiens et/ou des questionnaires en prenant appui sur les méthodes courantes des sciences sociales. L'entretien a, en général, la faveur des étudiants qui y trouvent à priori une manière plus simple d'aborder la question de la perception avec leurs interlocuteurs. Cet échange, enrichissant grâce à sa dimension dialectique, permet de cerner plus subtilement la question du sensible et de la subjectivité. À contrario, le question-
11. David Neau, Entre chien et loup, le crépuscule en photographie d'architecture, Énsa Nantes, 2011, 106 p. (mémoire soutenu avec l'équipe d'encadrement: P. Joanne, C. Marenne, T. Ouard).

12. Valentin Hardy, Quand le trottoir devient l'espace fumeur des bars, Énsa Nantes, $2010,96 p$.
13. Stéphanie Morio, L'écologie telle qu'on la voit, note méthodologique, juin 2010, 9 p.

Document interne.

14. Pauline Jadeau, Image nocturne du gratte-ciel, note méthodologique, juin 2010, non paginé. Document interne. 
naire relève d'un apprentissage plus long et nécessite un travail en amont plus important. Les données qui en sont issues sont plus complexes à analyser mais peuvent aussi compléter utilement les entretiens car les questions posées, et donc les réponses attendues, ne sont pas les mêmes. Néanmoins, la difficulté d'analyser des données statistiques et de mesurer plus finement la part de subjectivité dans l'analyse des ambiances, découragent souvent les étudiants qui préfèrent des méthodes plus exploratoires afin de mieux appréhender le sens des choses. Dans de plus rares cas, la méthode de l'écoute rétroactive permet de saisir comment une séquence sonore peut être perçue de manière différente selon la sensibilité et le vécu des auditeurs. En faisant écouter une séquence d'un parcours urbain en ville au bord d'une rivière, un étudiant a montré en quoi la perception des sons produits par la ville (trafic routier) et par la nature (oiseaux, eau) pouvait varier selon que les auditeurs étaient voyants ou non voyants, habitués des lieux ou étrangers, citadins ou ruraux ${ }^{15}$.

Dans le second cas, les mémoires qui partent de données déjà existantes portent sur des analyses comparatives selon un schéma scientifique classique. Certains mémoires partent d'une donnée statistique ( $x$ \% de Français préfèrent la maison individuelle, par exemple) pour poser un questionnement et construire une problématique de recherche. D'autres sont basés sur un corpus de nature iconographique (images, photographies, séquences de films) ou littéraire (textes) qui sert de matériau pour comprendre les phénomènes d'interprétation des ambiances. En interrogeant la référence en architecture comme producteur de sens pour le projet architectural, des modes et des outils de représentation sont analysés, ainsi que des discours ou des romans dans lesquels la question de l'ambiance est mise en perspective.

En somme, le déroulé de la méthode se résume en trois points : les outils de la méthode (questionnaires, entretiens, analyse comparative, etc.), la production de données et l'obtention de résultats. II n'est pas rare que les trois parties ne soient pas abouties, notamment au niveau des résultats dont l'obtention n'est jamais acquise à l'avance. La bonne méthode est celle qui ne cherche pas à venir corroborer les hypothèses de départ mais qui reste suffisamment neutre pour laisser émerger des réponses parfois inattendues. Dans le cas inverse, les résultats sont faussés. Un mémoire portant sur la perception de l'espace domestique par des personnes trisomiques ${ }^{16}$ a posé I'hypothèse qu'il existerait une différence de perception due à la différence génétique. En menant des entretiens, l'étudiante a été surprise d'aboutir à des résultats démontrant l'inverse. La réussite du mémoire de recherche a tenu ici dans la capacité de l'étudiante à avoir su construire une méthode adaptée pour obtenir des réponses objectives et des résultats venant infirmer l'hypothèse de départ.

La liberté qui est laissée à l'étudiant dans le choix d'adapter son outil d'investigation explique l'existence de questionnaires ou d'entretiens fermés, semi-fermés ou ouverts, dirigés ou non dirigés. C'est souvent dans la mixité de ces formes d'outils que l'équilibre est à trouver. "II est faux de croire que les recherches les plus rigoureuses sont celles qui font appel à des méthodes très formalisées et il est tout aussi faux de

15. Erwann Tessier, Lecture sonore de l'Erdre dans le paysage urbain de Nantes, mémoire de séminaire, Énsa Nantes, 2008, 101 p.

16. Céline Fésard, Trisomie 21 : les ambiances d'un chez-soi, mémoire de séminaire, Énsa Nantes, 2008, 128 p. 
penser qu'un chercheur ne peut faire preuve de rigueur qu'au détriment de son imagination ${ }^{17}$. " En travaillant sur l'importance du jardin dans I'habitat individuel ${ }^{18}$, une étudiante a mis au point un outil mixte (à la fois entretien et questionnaire : questionnaire dirigé) dans lequel à aucun moment le jardin n'est évoqué, afin de ne pas influencer les réponses. Cette méthode construite par l'absence de l'objet principal dans les questionnaires s'est révélée très concluante puisque les personnes interrogées en venaient d'elles-mêmes à citer le jardin comme élément d'usage conférant une qualité d'ambiance à ce type d'habitat.

Comme toute démarche scientifique, la recherche de résultats n'est pas garantie quand bien même le sujet, la problématique et la méthode sont clairement exposés ; la bonne méthode est celle qui ferme la boucle de la démarche de recherche en permettant de réinterroger la problématique, à l'aune des résultats obtenus ou non. L'absence de résultats n'est pas considérée comme un échec du moment que la méthode est au point. Plusieurs circonstances peuvent expliquer l'absence de résultats concrets, notamment l'insuffisance de l'échantillonnage des personnes interrogées. Pour autant, si la problématique réfère bien à un questionnement construit autour d'une méthode de recherche et que les arguments développés sont solides, l'équipe pédagogique considère que la mission est remplie. L'objectif du mémoire réside dans la construction de la démarche de recherche; en cela la mise au point de la méthode est fondamentale et c'est en quoi l'exercice du mémoire de recherche est formateur. À ce niveau, la production de connaissances reste secondaire en regard de la compréhension et de la mise en œuvre du mécanisme de recherche.

L'initiation à la recherche vise un double objectif : elle permet, d'une part, d'apprendre et de mettre en œuvre des méthodes appropriées et, d'autre part, de comprendre qu'un sujet reste inerte s'il n'est pas interrogé de manière dynamique. L'ouverture à des dimensions supplémentaires d'introspection du projet architectural et urbain, est très profitable à la maturation intellectuelle de l'étudiant et à sa capacité de maintenir une distance critique vis-à-vis de son environnement.

Les ambiances peuvent être le vecteur de ces questionnements. La grande hétérogénéité de ses champs disciplinaires, de ses outils et de ses méthodes d'analyses, toujours à réinventer, en fait un objet de recherche à part entière. Son périmètre mouvant, sans cesse redéfini et constamment réinterrogé, est un puissant stimulant à l'exploration de nouveaux questionnements et à des mises en perspective originales. La " jeune recherche en architecture », aussi néophyte dans les manières de chercher que dans l'appréhension de la notion d'ambiance gagne, avec cette expérience, une compétence nouvelle.

17. Raymond Quivy, Luc Van Campenhoudt, Manuel de recherche en sciences sociales, Paris, Dunod, 1995, p. 238.

18. Sabine Lepère, De la maison individuelle au logement collectif. Le jardin privé peut-il permettre de concilier les aspirations des ménages avec les préoccupations actuelles de densité urbaines? Énsa Nantes, 2010, 77 p. 